Ann. Biol. anim. Bioch. Biophys., 1977, 17 (4), 543-547.

\title{
Utilisation de la gouttière œesophagienne pour éviter la dégradation dans le rumen, de mélanges de lactose et de caséine, chez le veau ruminant
}

\author{
par R. GUILHERMET, P. PATUREAU-MIRAND*, R. TOULLEC, J.-L. PARUELLE ** \\ avec la collaboration technique de Marguerife BEAUFILS et P. GAUCHET \\ Station de Recherches Zootechniques, I. N. R. A., \\ Ecole Nationale Supérieure Agronomique, \\ 65, rue de Saint-Brieuc, 35042 Rennes Cedex. \\ * Laboratoire d'Etudes du Métabolisme Azoté, I. N. R. A., \\ Theix, Saint Genès Champanelle, 63110 Beaumont. \\ ** Union des Coopératives d'Aliment du Bétail \\ BP 75, 02400 Chateau-Thierry.
}

Summary. Effect of by-passing the rumen with supplements of casein and lactose in young ruminant calves.

Fourty-eight Frisian calves were weaned at the age of 7 weeks. They were distributed into 4 groups one week before weaning. Thereafter, for an experimental period of 8 weeks, the animals received 4 diets composed of hay (15 p. 100) and experimental concentrates (85 p. 100). The latter contained mixtures of cereals and soybean $(80$ p. 100) and supplements (20 p. 100) with casein and deproteinized whey (table 1$)$. These supplements were offered either solid (introduced into the experimental concentrate) or liquid (in a trough with $240 \mathrm{~g}$ dry matter in $760 \mathrm{~g}$ water ; one meal in the morning). Moreover, 2 levels of protein were used in the diets for each mode of feeding. The dry matter supply increased from 22 to $77 \mathrm{~g} / \mathrm{kg}$ live weight 0,75 between the ages of 7 and 14 weeks. Jugular blood samples were taken at the ages of 8,10 and 14 weeks, 4 hours after the morning meal. Feeding the supplements in liquid form led to a large increase $(P<0,01)$ in the plasma level of glucose irrespective of the protein level (fig. 1). It also brought about a decrease in the plasma level of urea $(P<0,01)$, after the age of 8 weeks (fig. 2). Thus, the supplements when fed liquid, arrived directly in the abomasum and avoided degradation in the rumen; so, they were absorbed as glucose, galactose and amino-acids, instead of being fermented as lactic acid, volatile fatly acids and ammonia. The amount of aminoacids absorbed was most likely improved. However, no important increase in the blood levels of most of the essential amino acids was observed, contrary to our previous results where a larger part (52 p. 100) of the protein was fed in liquid form. Even the blood levels of valine, leucine and isoleucine diminished. This is probably due to the great quantity of glucose absorbed with liquid supplements, which would have improved metabolic amino acid utilizalion. Consequently, growth and feed efficiency were significantly improved with the liquid feeding. 
Des travaux précédents (Guilhermet, Patureau-Mirand et Toullec, 1976) ont mis en évidence une amélioration de l'état nutritionnel des veaux ruminants en utilisant la forme liquide pour faire passer un supplément riche en protéines de soja directement dans la caillette. Cependant, la faible quantité d'énergie ingérée par le jeune veau ruminant jusqu'à 3 mois, ne lui permet pas de valoriser par une meilleure croissance les acides aminés mis ainsi à sa disposition. Aussi, il nous est apparu opportun d'adjoindre une source d'énergie au supplément protéique pour comparer les formes solide ef liquide de distribution. La caséine a été choisie comme source de protéines et le lactose comme source d'énergie.

48 veaux mâles frisons ont été sevrés à l'âge de 7 semaines. Une semaine avant le sevrage, ils ont été divisés en 4 lots de 12 animaux et ont commencé à recevoir 4 régimes comprenant 15 p. 100 de foin et 85 p. 100 d'aliments expérimentaux. Ces derniers étaient constitués pour 80 p. 100 par des mélanges de céréales $c t$ de tourteau de soja, et pour 20 p. 100 par des suppléments de caséine et de lactosérum déprotéiné par ultrafiltration. Les suppléments ont été distribués sous forme solide (incorporés dans le mélange de céréales + tourteau) ou liquide (un repas par jour au seau, à

\section{TABLEAU 1}

Consommation, croissance, efficacité alimentaire et aminoacidémie libre (durée de la période expérimentale : 8 semaines)

Feed intake, growth, feed efficiency and blood level of free aminoacids (length of the experimental period : 8 weeks)

\begin{tabular}{|c|c|c|c|c|}
\hline \multirow{2}{*}{$\begin{array}{l}\text { Forme des suppléments * } \\
\text { Niveau protéique }\end{array}$} & \multicolumn{2}{|c|}{ Solide } & \multicolumn{2}{|c|}{ Liquide } \\
\hline & haut & bas & haut & bas \\
\hline 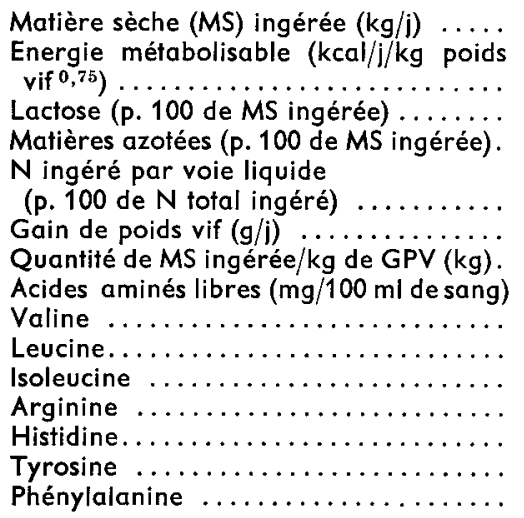 & $\begin{array}{c}1,59 \\
170 \\
10,8 \\
18,5 \\
- \\
643^{\mathrm{a}} \pm 100 \\
2,58^{\mathrm{a}} \pm 0,43 \\
2,03 \\
1,71 \\
1,14 \\
0,68 \\
1,36 \\
0,84 \\
0,83\end{array}$ & $\begin{array}{c}1,59 \\
173 \\
10,7 \\
15,3 \\
-641^{\mathrm{b}} \pm 69 \\
2,63^{\mathrm{bc}} \pm 0,43 \\
= \\
= \\
= \\
=\end{array}$ & $\begin{array}{c}1,64 \\
174 \\
11,7 \\
16,8 \\
23,0 \\
734^{a b} \pm 69 \\
2,23^{a b} \pm 0,13 \\
1,54 \\
1,31 \\
0,79 \\
0,83 \\
1,45 \\
1,04 \\
0,81\end{array}$ & $\begin{array}{c}1,63 \\
175 \\
11,6 \\
15,4 \\
23,5 \\
694 \pm 48 \\
2,38^{\mathrm{c}} \pm 0,17 \\
= \\
= \\
= \\
= \\
=\end{array}$ \\
\hline
\end{tabular}

* Mélanges de poudre de lactosérum déprotéiné $(75$ ef 79 p. 100) et de caséine (25 et 21 p. 100) respectivement pour les hauts et les bas niveaux azotés.

Mixture of deproteinized whey powder (75 and 79 p. 100) and of casein (25 and 21 p. 100) for high and low nitrogen levels respectively.

a, b, c : les valeurs de la même ligne, accompagnées des mêmes lettres, sont significativement différentes $(P<0,05)$. 
raison de $240 \mathrm{~g}$ de matière sèche pour $760 \mathrm{~g}$ d'eau). En outre, deux niveaux azotés ont été utilisés dans les régimes pour chacune des deux formes de présentation (tabl. 1). Les quantités de matière sèche proposée ont augmenté progressivement de 22 à $77 \mathrm{~g} / \mathrm{kg}$ de poids vif ${ }^{0,75}$, de la $7 \mathrm{e}$ à la $14 \mathrm{e}$ semaine d'age. Des prises de sang ont été effectuées dans la veine jugulaire à l'âge de 8,10 et 14 semaines, $4 \mathrm{~h}$ après le repas du matin.

Les animaux ont ingéré des quantités de matière sèche et d'énergie métabolisable voisines (tabl. 1). La glycémie a été plus élevée lorsque le supplément a été distribué sous forme liquide, indépendamment du taux protéique (fig. 1). La différence a été faible à la 8 e semaine d'âge ; cela peut s'expliquer par la faible quantité de supplé-

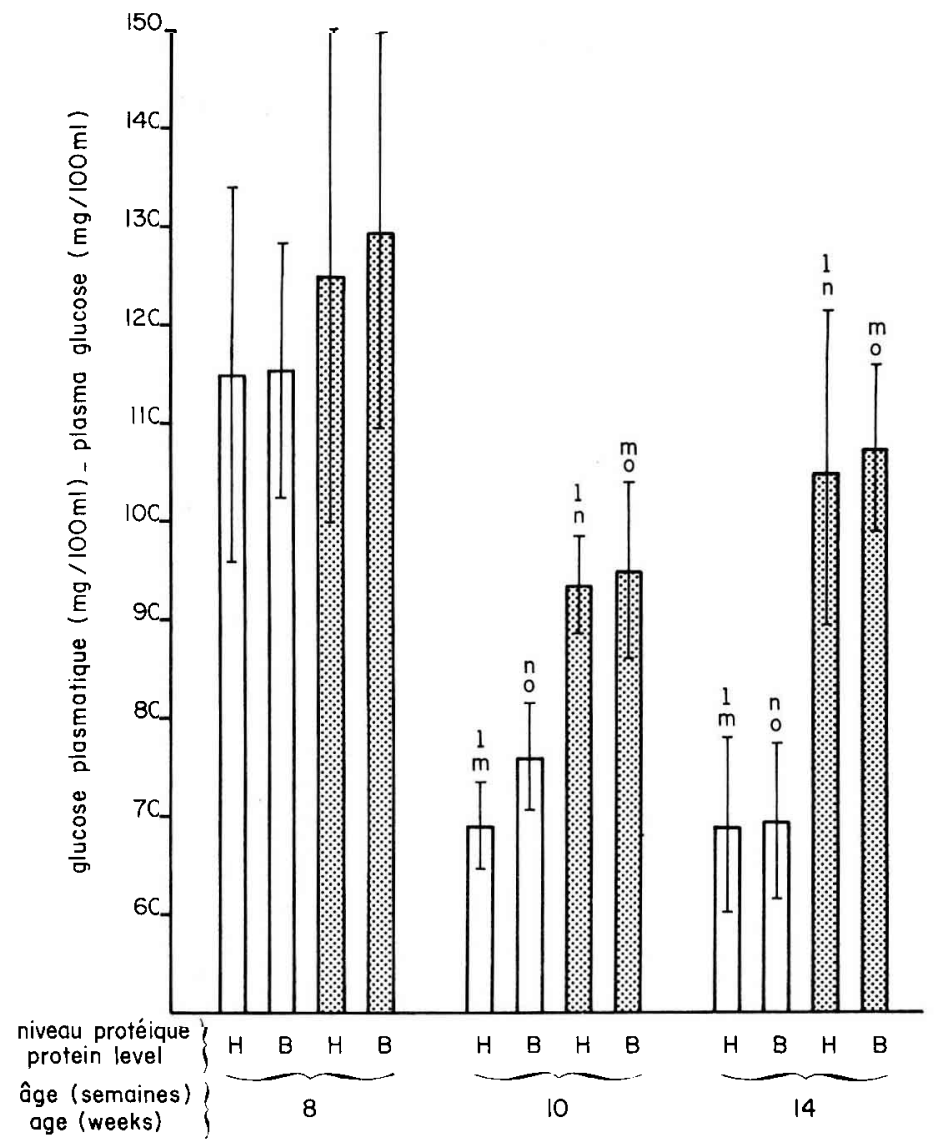

FIG. 1. - Influence de l'âge et du régime sur la glycémie.

$H=$ high $\square$ Supplément solide.

$B=$ low Supplément liquide.

(im) pour un âge déterminé, les valeurs accompagnées des mêmes lettres sont significativement différentes $(P<0,01)$. 
ment ingéré ( $240 \mathrm{~g}$ de MS soit $180 \mathrm{~g}$ de lacłose). En revanche, elle a été importante $(P<0,01)$ ensuite.

Après la 8 e semaine d'âge, l'urémie a été influencée à la fois par le taux azoté des régimes et par la forme de distribution du supplément (fig. 2). Elle a été plus

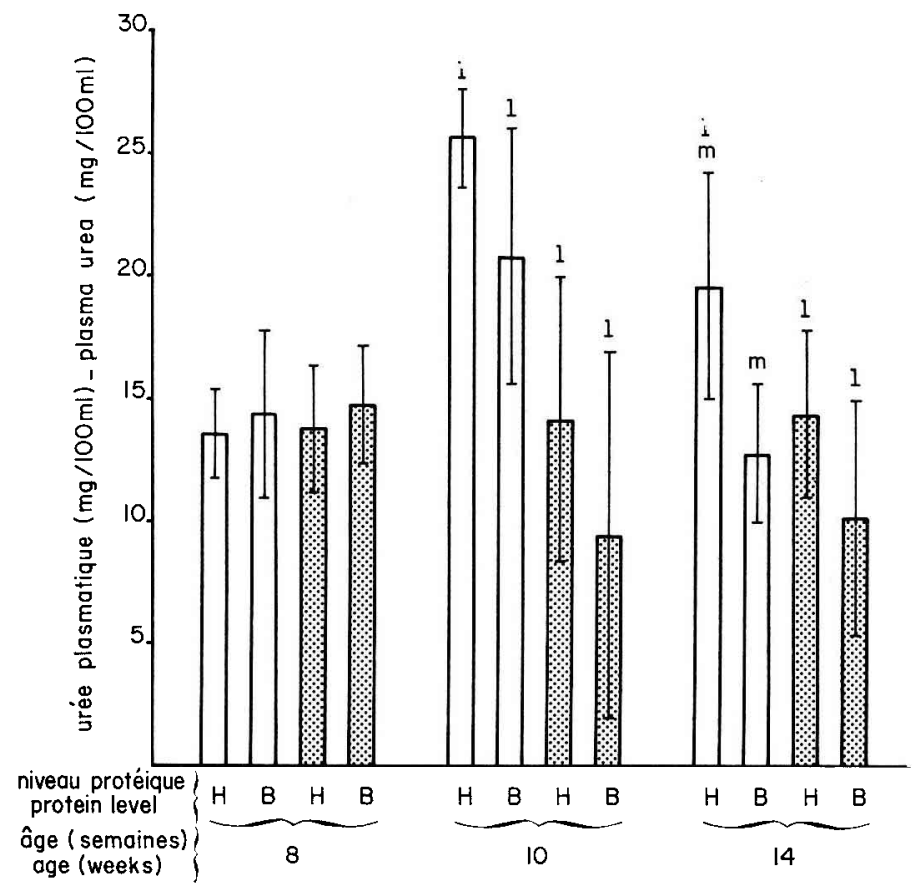

FIG. 2. - Effet de l'âge ef du régime sur l'urée plasmatique.

$\mathrm{H}=$ high $\square$ Supplément solide.

$B=$ low Supplément liquide.

$(\mathrm{lm})$ pour un âge déterminé, les valeurs accompagnées des mêmes lettres sont significativement différentes $(P<0,01)$.

faible $(P<0,01)$ avec la forme liquide, ainsi qu'avec le bas niveau azoté. La forme liquide a donc permis aux suppléments de passer directement dans la caillette (Guilhermet, Mathieu et Toullec, 1975) et d'être absorbés sous forme de glucose, de galactose et d'acides aminés, au lieu d'être dégradés dans le rumen en acide lactique, acides gras volatils et ammoniaque. La quantité d'acides aminés absorbés a probablement été augmentée. De plus, la caséine est plus riche en la plupart des acides aminés indispensables, en particulier en histidine et méthionine, que les matières azotées du contenu de duodénum de bovin recevant un régime riche en aliment concentré (Champredon, Pion et Thivend, 1971). Cependant, la somme des acides aminés indispensables + tyrosine libres du sang a été plus faible avec la buvée $(9,8$ au lieu de 10,7 pour $100 \mathrm{~g}$ de sang) (tabl. 1), contrairement à ce que nous avions noté au 
cours d'un autre essai, en distribuant une part plus importante (52 p. 100) des protéines alimentaires sous forme liquide (Guilhermet, Patureau-Mirand ef Toullec, 1976). La diminution a porté particulièrement sur la valine, la leucine ef l'isoleucine, vraisemblablement à la suite de l'absorption de quantités importantes de glucose par les veaux recevant la buvée ; Eskeland, Pfander ef Preston (1974) ont observé le même phénomène en infusant $d u$ glucose à des agneaux. Cela pourrait s'expliquer par un accroissement de la sécrétion d'insuline sous l'afflux de glucose ou d'acides aminés (Faichney et Weston, 1971 ; Basset, 1975). L'arginine est le seul acide aminé à être notablement plus élevé dans le sang des veaux soumis à la buvée, alors que la quantité absorbée a dô être inférieure. Il est probable que cela correspond à une moindre activité du cycle de l'urée (arginase en particulier) chez les animaux donf l'urémie est plus faible, sans doute du fait d'une moindre ammoniogénèse dans le rumen. Cela paraît confirmé par les teneurs plus faibles en ornithine chez les veaux ayant reçu la buvée $(0,65$ au lieu de $0,53 \mathrm{~g}$ pour $100 \mathrm{~g}$ de sang).

L'apport de protéines de bonne qualité (bien que légèrement déficientes en acides aminés soufrés) et d'énergie (glucose) sous forme de buvée, semble s'être accompagné d'une augmentation de l'utilisation métabolique des acides aminés absorbés. II en a probablement été de même pour le rendement de l'énergie métabolisable. En conséquence, la croissance ef l'efficacité alimentaire ont été plus satisfaisantes chez les veaux des lots qui ont reçu les suppléments sous forme liquide (tabl. 1).

Commission CNERNA Digestion-Absorption, Tours, 13 novembre 1976.

\section{Références}

BASSET J. M., 1975. Dietary and gastro intestinal control of hormones regulating carbohydrate metabolism in ruminants. In Mc DONALD J. W., WARNER A. C. I., Digestion and metabolism in the ruminant, 383-398. The University of New-England Publ.

CHAMPREDON C., PION R., THIVEND P., 1971. Composition en acides aminés des matières azotées du contenu duodénal du ruminant. $X^{\mathrm{e}}$ Congr. Infern. Zoofech., Versailles.

ESKELAND B., PFANDER W. H., PRESTON R. L., 1974. Intravenous energy infusion in lambs : effects on nitrogen retention, plasma free amino acids and plasma urea nitrogen. Brit. J. Nutr., 31, 201-211.

FAICHNEY G. J., WESTON R. H., 1971. Digestion by ruminant lambs of a diet containing formaldehyde treated casein. Aust. J. agric. Res., 22, 461-468.

GUILHERMET R., MATHIEU C. M., TOULLEC R., 1975. Transit des aliments liquides au niveau de la gouttière œesophagienne chez le veau préruminant et ruminant. Ann. Zootech., 24, 69-79.

GUILHERMET R., PATUREAU-MIRAND P., TOULLEC R., 1976. Influence de la distribution sous forme solide ou liquide d'un supplément riche en protéines chez le veau ruminant. Ann. Zootech., 25, 281-286. 